Voix et Images

voixetimages

\title{
Jacques Godbout, diariste
}

\section{Yvon Bellemare}

Volume 10, numéro 3, printemps 1985

André Major

URI : https://id.erudit.org/iderudit/200521ar

DOI : https://doi.org/10.7202/200521ar

Aller au sommaire du numéro

Éditeur(s)

Université du Québec à Montréal

\section{ISSN}

0318-9201 (imprimé)

1705-933X (numérique)

Découvrir la revue

Citer cet article

Bellemare, Y. (1985). Jacques Godbout, diariste. Voix et Images, 10(3), 152-164.

https://doi.org/10.7202/200521ar d'utilisation que vous pouvez consulter en ligne.

https://apropos.erudit.org/fr/usagers/politique-dutilisation/ 


\title{
Jacques Godbout, diariste
}

\author{
par Yvon Bellemare, Collège Mérici
}

Jacques Godbout dans son Journal d'hiver1, texte composé entre décembre 1981 et avril 1982, n'hésite pas à déclarer que l'écrivain «s'écrit» dans ses textes. Cette affirmation revient à dire que, dans son œuvre et en particulier dans ses romans, Godbout exprime aussi le sujet qu'il est d'une certaine façon, son Je, lorsqu'il imagine le thème d'un livre. En effet, selon l'expression d'Alain Goulet, l'œuvre est une «machine à exprimer un moi2». Que ce Je soit mis à nu comme dans Souvenirs pour demain 3 de Paul Toupin, ou maquillé et camouflé sous l'identité d'un auteur-narrateur-personnage4, il n'en demeure pas moins que les narrateurs des romans godboutiens, tels des masques savamment moulés, figurent le cheminement personnel de leur auteur. L'imaginaire de la fiction offre la possibilité de dessiner les différents contours d'une personnalité et même d'exprimer, consciemment ou non, les données chères au créateur de l'œuvre. La désarticulation de quelques instances des registres 5 dans les romans de Godbout et le Journal d'hiver permet de démasquer un Moi se retranchant derrière une technique qui auto-génère une multitude de $J e$ difficiles à saisir dans leur totalité.

\section{L'apparence taxinomique}

Plusieurs récits de Godbout se classent sous l'apparence taxinomique du journal intime: «Sorte de reportage plus ou moins immédiat ${ }^{\text {» }}$ de faits qui touchent de près le narrateur. Comme les pseudo-journaux intimes du Journal d'un curé de campagne, de la Nausée ou du Journal d'un fou, les romans godboutiens, de Salut Galarneau! 7 aux Têtes à Papineau8, présentent les indices d'une fiction où les narrateurs enregistrent régulièrement les événements qu'ils vivent 9 , autrement dit «l'accent porte sur les relations du narrateur avec l'événement $10 \%$. Cette formule autobiographique que privilégie Godbout est une sorte de pastiche au sens où l'entend Genette, à savoir que l'écrivain «n'imite pas un texte mais un stylell». L'hypertextualité des romans de Godbout n'apparaît donc pas seulement comme un simple exercice. Sa répétition soutenue oblige à croire que cette imitation facilite la relation de l'auteur avec l'art, ou ce que l'on pourrait appeler sa «cosmogonie», c'est-à-dire son univers aussi bien réel que purement romanesque. C'est d'ailleurs ce qu'affirme le diariste dans les premières lignes de son Journal d'hiver: «L'écrivain, s'il avance quelque angoisse, ne le fait que pour mieux la partager 12).

Examinons d'abord les caractéristiques du Journal d'hiver. Les quelque quatre mois que résume ce journal intime braquent l'objectif sur des faits qui ont touché directement le Je exprimant sa surprise, son mécontentement ou tout simplement sa satisfaction. On se rend vite à l'évidence que Godbout concentre toute son énergie sur ce qui, à certains jours, l'a agacé ou profondément intéressé. L'écriture spontanée, l'immédiateté de chacune 
des idées, permettent de constater que Godbout ne s'enferme guère dans des conclusions définitives. Au contraire, cet exercice d'écriture est comme au diapason de la saison d'hiver 81-82 qui n'en finit pas: elle s'étire dans l'attente d'un peu plus de chaleur13. Mais ce journal entrepris par Godbout n'est pas un monologue qui tourne à vide, cela se veut plutôt un dialogue qui s'établit entre un auteur et son public. En effet, Godbout s'y présente comme un écrivain qui va du dramaturge au conférencier, du romancier au cinéaste, de l'éditorialiste au poète, et dont le but ultime est d'alimenter la discussion, de secouer les léthargies, de faire des propositions 14. Les multiples sujets abordés ressemblent aux morceaux d'une mosaïque, qui essaient de former un tout cohérent malgré l'apparent désordre. Ce qui compte d'abord, c'est de faire ressortir l'importance du moment présent, celui où Godbout ressent les rigueurs de l'hiver et traque les bizarreries de l'existence. Cette conception entre bien dans les vues de Peter Boerner qui affirme que «la présentation du journal intime met l'accent non plus sur la continuité dans le temps mais sur le présent $15 »$.

L'architextualité du Journal d'hiver est une évidence dont ne profitent pas les autres récits de Godbout. En effet, même si plusieurs romans godboutiens reposent sur le principe structurel du journal intime, ce n'est là qu'un cadre qui emprunte certaines caractéristiques du genre. Les vingtquatre chapitres de l'Aquarium 16 transcrivent au jour le jour les déboires d'un Je noyé dans l'indifférence et l'insouciance d'une Casa perdue quelque part, ce qui permet au narrateur une introspection qui l'empêche de sombrer dans le désespoir. Découpé en quatre-vingt-cinq fragments d'inégale longueur, le deuxième roman, le Couteau sur la-table17, livre à son tour un $J e$ en proie à la révolte devant le conquérant. Les différentes pièces qui forment ce récit favorisent une réflexion sur le passé, les amours et surtout l'appartenance du protagoniste à une nation vaincue. Même si ces deux romans ne possèdent pas d'indications temporelles, comme le veut ordinairement le journal intime, ils retiennent toutefois des éléments essentiels de ce genre: la réflexion dûment entretenue et une grande liberté d'expression. En revanche, Salut Galarneau! emprunte davantage à la structure "visible» du journal intime, car François «vécrit» ses deux cahiers comme le ferait tout diariste consciencieux. C'est du reste ainsi qu'il découvre la fascination de l'écriture. Quant à l'Isle au dragon 18, il imite le journal intime plutôt dans sa forme et son contenu. Échelonné sur sept jours, ce roman reprend l'angoisse d'un Beauparlant qui doit affronter la menace que fait courir à l'environnement l'enfouissement de déchets atomiques dans son «isle». D'un lundi à l'autre, on suit le narrateur qui s'interroge, rouspète, s'engage et coiffe au poteau son ennemi. Enfin, les Têtes à Papineau, récit «bigraphique» que Charles et François écriront ad finem, présente "tout

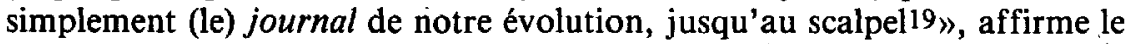
double narrateur. François précise de son côté que son aventure avec Charles offre l'originalité d'un «journal à deux voix 20 ». Divisé en' huit sections suivies d'un "enfin» composé séulement par Charles, cet écrit relate la transformation de Charles-François Papineau, depuis la naissance en 1955 jusqu'à l'intervention finale du Dr Northridge. 
Il n'y a qu'un pas entre le journal intime qui s'écrit régulièrement et l'écriture journalistique propre aux quotidiens 21 . Godbout le franchit allégrement, et avec d'autant plus d'aisance qu'il s'alimente à toute forme d'actualité. Plusieurs sujets, en effet, s'inspirent de la presse journalière: l'internationalisme et la décolonisation dans l'Aquarium; la problématique nationaliste de l'impossible alliance du "Canadian-Québécois» dans le Couteau sur la table et dans les Têtes à Papineau; la découverte de la magie des mots dans Salut Galarneau!, la libération de la femme dans D'amour, $P . Q .22$; et enfin la menace que fait peser sur la nature l'ère atomique dans l'Isle au dragon. Le journal, image même du divers et du multiple, permet aux romans de Godbout de s'amuser avec un collage de nouvelles aussi surprenant qu'insolite, alors qu'en parallèle s'inscrit un texte où brille la poésie. «Et quoi d'étonnant?, soutient Gilles Marcotte, puisque formellement rien ne ressemble plus à un journal qu'un poème d'après Baudelaire, rompant avec la phrase et le sens continu au profit de la mosaïque verbale 23 ».

En imitant la structure du journal intime et en retenant certains mécanismes propres au journalisme, l'auteur-narrateur-personnage figure un être réflexif qui, en écrivant, vit. François Galarneau résume bien cette manière-d'être-au-monde lorsqu'il avoue au policier Alfred: "Tu sais, Alfred, quand on écrit quelque chose qui nous est arrivé, c'est comme si on le vivait une fois encore avec toutes les émotions, presque...24». Cette feinte romanesque favorise la confidence de l'auteur, qui s'ouvre ainsi au lecteur par le biais de ses héros, et il le fait avec d'autant plus de facilité qu'il se cache sous le voile du narrateur. L'auteur s'introduit dans l'ouvrage pour en régler l'orientation, tandis que le narrateur joue le rôle du producteur d'écriture, grâce à l'hypertextualité du journal intime.

\section{Les doublures du Je}

Dans un deuxième temps, il sied d'explorer le statut du narrateur, à savoir qui parle. Quel statut possède celui qui raconte par rapport à ce qui est raconté? C'est l'examen des différents niveaux de représentation du narrateur incarnant le sujet de l'énonciation, qui permettra de découvrir l'originalité de Godbout lorsqu'il exploite les multiples moyens qu'offre l'instance discursive.

Quand l'Aquarium paraît en 1962, Jacques Godbout est revenu depuis quelque temps déjà d'un séjour de trois ans comme professeur de philosophie, puis de français, à l'University College d'Addis-Abeba en 'Éthiopie. Ce premier roman n'est pas une autobiographie, certes, mais les interrogations du Je de la Casa Occidentale, comme ses prises de position, ne sont pas indépendantes du cheminement de l'auteur. En effet, à travers le personnage principal transpire page après page une atmosphère que Godbout n'a pu ignorer: le régime finissant et toujours autoritaire de Maurice Duplessis. Les comportements parfois curieux du Je de l'Aquarium, sa lâcheté, sa mesquinerie, voire son indifférence face à une exploitation oc- 
cidentale démesurée, le conduisent pour ainsi dire à profiter d'une occasion inattendue, l'arrivée d'Andrée, pour fuir un milieu où l'ennui, la solitude, l'escroquerie et le détournement des intentions au profit du plus fort, dégradent un peu plus chaque jour l'individu.

Au début de ce'roman, le narrateur identifié surtout à un $J e$, s'impose même s'il a peur de ses responsabilités. Toutefois, ce premier Je se métamorphose en Nous ou en $I l$, façon de se réfugier dans le collectif et l'impersonnel. Le $J e$ comme le $I l$ permettent des incursions où l'indiscrétion joue un rôle important. L'omnicience du Je de l'Aquarium dresse facilement l'inventaire des faits et gestes des personnages, même s'il n'est pas présent. Les séquences oủ Monsignore palpe et caresse l'argent qu'il vient de voler25, n'échappent pas à la clairvoyance du $J e$, qui se complaît aussi à détacher du noir de la nuit les moindres déplacements du prélat. se rendant chez les putains 26 . Bien plus, ce Je détecte les pensées des personnages. Un exemple parmi tant d'autres illustre cette capacité de divination: le narrateur sait que Gayéta a hésité face au comportement de ce dernier, mais il ajoute: "Je sais, je sens qu'il va revenir27». Enfin, le Je omniscient se glisse dans le for intérieur des personnages et décrypte leurs rêves et leurs souvenirs. La vocation missionnaire de Pauline et de Monsignore est dévoilée au lecteur grâce à l'étrange connaissance dont jouit le narrateur qui a su pénétrer dans l'intimité du cloître où «Pauline a rencontré Monsignore qui revenait des missions 28 ".

Le Je de l'Aquarium, comme une sorte d'ectoplasme, hante le destin de chaque personnage. Les trois premiers chapitres de ce roman lui sont totalement confiés. Il y relève les vices de Monsignore et les préparatifs des indigènes en vue de l'insurrection. À plusieurs reprises, Je surprend le prélat alors qu'il patauge dans la turpitude. L'acharnement du narrateur à dénoncer les tricheries du missionnaire suggère que Monsignore incarne aux yeux du $J e$ tout ce qu'il abhorre: l'exclusivité d'une vérité douteuse et la malhonnêteté cachée29.

Au Je omniscient de l'Aquarium succède, à maintes occasions, un Je non identifié. Dans ces passages-là, force est de chercher un autre narrateur, «car non seulement il s'agit d'actes dont le peintre canadien n'est pas témoin, mais parfois un mot ironique a bien l'air d'un jugement sub specie aeternitatis 30 ". Godbout, en effet, s'introduit dans l'histoire et joue à l'auteur omniscient qui a du mal à retenir sa malice. Ces apparitions surprenantes de l'auteur engendrent des invraisemblances. La description des prouesses de Monsignore, l'enregistrement à l'Administration de la mort de Lui ou la révélation du passé de Pauline représentent des exemples typiques du narrateur qui se faufile dans l'intimité de ses personnages.

Le $J e$ du premier roman de Godbout, ramassé sur lui-même et comme ratatiné dans sa médiocrité, est remplacé dans le Couteau sur la table par un $J e$ qui préfère humer l'étendue des grands espaces. On ne peut ignorér que le narrateur, jeune Canadien français au passé entaché de plus d'une fredaine, cherche une partenaire qui pourrait lui procurer une certaine sécurité affec- 
tive et même matérielle. L'ambivalence de ce Je tiraillé entre Patricia, l'anglophone, et Madeleine, la compatriote francophone, est une figure pouviant facilement se comparer à celle du bicéphale Charles-François des Têtes à Papineau. En effet, la portée nationaliste de ces deux romans n'a pas à être démontrée, elle serait plutôt à exploiter afin de confirmer ce que Godbout soutient dans son Journal d'hiver: le Canada n'est pas un pays, c'est plutôt un contrat 31 . Car la liaison de $J e$ avec Patricia, un francophone et une anglophone, et les liens entre la tête anglaise de Charles et la tête française de François, illustrent sans équivoque la fragilité, voire l'aberration du «deux dans un», deux nations dans un seul pays, que la mère du bicéphale, Marie Lalonde, s'entête à refuser.

Dans le Couteau sur la table comme dans les Têtes à Papineau, Jacques Godbout dresse un réquisitoire contre l'ambivalence du Canadien français. D'abord, le Je du Couteau, déchiré entre Patricia et Madeleine, l'Ouest et l'Est, vit des scénarios où la rupture est monnaie courante. Il en est ainsi parce qu'il semblerait qu'aucun lien permanent ne paraisse viable. Godbout va plus loin. Son cheminement personnel, son Je profond qui ressemble parfois étrangement à celui de son roman, marque pour ainsi dire une rivalité latente entre une Patricia cossue, emblème du colonisateur, et un narrateur totalement dans l'insécurité, ombre portée de tout colonisé. Commencé sous le signe de la bonne entente entre les «deux solitudes», le récit se termine avec l'image d'un couteau sur la table, signe concret d'une mésentente. L'idylle amoureuse entre l'anglophone et lui-même tourne court. La fin métaphorique de ce roman relève sûrement de la transposition d'un sentiment ou d'un état d'être que vivait alors Godbout32. La menace finale que le narrateur Je profère contre Patricia rompt définitivement toute alliance possible entre les solitudes anglaise et française au Canada.

Par ailleurs, le Je du Couteau sur la table s'est métamorphosé en un Nous, celui du bicéphale, dans les Têtes a Papineau. Les deux solitudes du premier se sont comme «amalgamées» provisoirement. Le bicéphale que met au monde une Marie Lalonde surprise et fière, reprend à sa façon les contradictions inhérentes à une situation qui trouble Jacques Godbout. Cette entité juridique qu'est le Canada suscite plus d'un débat aboutissant à des décisions qui, inévitablement, briment l'une des parties en cause. De 1965 jusqu'en 1980, de la violence du F.L.Q. aux diatribes épiques qui ont marqué le référendum québécois, Jacques Godbout n'a cessé de décrier l'aberration du système canadien. Aussi ce récit illustre-t-il la situation politique du peuple québécois tiraillé entre une francophilie bien légitime et une anglomanie qui a son utilité. Godbout transpose donc dans les Têtes à Papineau la confrontation légendaire d'un peuple qui a du mal à choisir politiquement ce à quoi il aspire réellement au plan culturel, économique et social.

L'ambivalence du tandem narrateur des Têtes à Papineau met en relief les activités et les goûts du couple, entendu ici dans l'acception que donne à ce mot la physique. Du reste, chacun d'eux possède un tempérament qui n'est pas étranger à son prénom et les deux composantes ne sont pas sans 
refléter la tendre enfance du romancier. En effet, né d'un père francophone de la paroisse Saint-Éloi sur l'Isle-Verte et d'une mère native de SainteAnne de Bellevue, Godbout, dès ses quatre ans, a dû apprendre l'anglais pour communiquer avec une jeune voisine amie lorsqu'il habitait Côte-desNeiges. Toutefois, il se place volontiers sous le patronage de François qui, comme dans Salut Galarneau!, fait revivre tout ce qui rappelle la vieille France avec sa langue savoureuse et ses traditions. Cependant, il n'ignore pas le Charles qui est tapi en lui et qui partage des affinités surtout avec la pensée anglaise et américaine. Bien entendu, Godbout évite de parler directement du Moi double qui l'habite, car il a horreur de l'étalage sur la place publique, mais le lecteur averti ne peut éviter de songer à la situation que vit l'auteur. Tel un juge, le bicéphale relève les travers et les ambivalences parfois criantes des $\mathrm{Moi}$ «juxtaposés», et cela avec plus de netteté que dans le Couteau sur la table.

À mi-chemin du Je déchiré entre Patricia et Madeleine et du Nous du monstrueux duo Charles-François, se placent François Galarneau et Michel Beauparlant. Le premier comme le dernier s'inspirent largement du quoti-: dien retenu par Godbout.

Salut Galarneau! est avant tout le fruit d'une réaction à la morbidité d'Une saison dans la vie d'Emmanuel33 de Marie-Claire Blais, de sa complaisance pour les Jean-Le-Maigre éternellement petits et écrasés. Le personnage de François, le Je écrivant, s'inscrit dans la lignée des créateurs appelés à surmonter des difficultés multiples avant de parvenir enfin à une espèce de libération productrice. Les traîtrises de Louise Gagnon et de Marise Doucet conduisent François à la VÉCRITURE, c'est-à-dire à l'utilisation, dans ses propres cahiers, de sa vie et de son milieu dûment intégrés dans un esprit créateur et libérateur. En effet, vivre et écrire, actes désormais inséparables, nient de façon impérative tout emmurement dans l'écriture pour elle-même. C'est le refus d'en faire une sorte d'abstraction. L'occupation libératrice qu'est l'écriture, ancrée dans le quotidien, en plus' de rendre autonome le $J e$, engendre la libération qui permet pour ainsi dire une seconde vie. Cette réinvention de la vie assurée par les mots, nouvelle manière pour Godbout de s'approprier l'activité littéraire, ne se confine plus, comme dans l'Aquarium et le Couteau sur la table, à un mécanisme reproducteur du passé, mais s'engage dans une perspective ouverte sur l'avenir.

$D$ 'amour, $P . Q$., sorte de synthèse de la décennie 60-70, de l'ère duplessiste à l'époque péquiste, imagine un auteur qui travaille à plusieurs manuscrits. Comme le Je écrivant de Salut Galarneau! qui transforme en écriture ses déboires, «l'auteur» de D'amour, P.Q. travaille pour sa part à confectionner des textes. Il «joue à l'écrivain 34 ». L'influence de Mireille, sa secrétaire, change peu à peu la langue utilisée. À la fin, en rejetant l'autorité d'une culture étrangère, le narrateur-auteur s'engage politiquement et répand des communiqués nationalistes qui ressemblent à ceux que le F.L.Q. lançaient sur les ondes de la radio et de la télévision. Encore ici, Godbout; visiblement bouleversé par ce qui se passait dans son milieu, élève au niveau 
littéraire des documents empreints de violence. La réalisation du projet national coïncide alors avec le projet littéraire.

Pour les narrateurs de Salut Galarneau! et de D'amour, P.Q., écrire est un acte positif. Le procédé dialectique du VÉCRIRE d'une part, la destruction de l'écriture et sa reconstitution d'autre part, aboutissent à une recherche sur l'expression qui permet l'identification des valeurs propres à la société québécoise. C'est là une prise de conscience affranchissante du Je québécois puisant dans l'imaginaire collectif qui l'anime.

Enfin, propriétaire d'un lopin de terre sur l'Isle Verte en face de Rivière-du-Loup, Godbout utilise encore une fois le roman pour s'opposer farouchement aux désirs illimités des multinationales, de même qu'à l'envahissement désordonné d'une civilisation américaine où la consommation excessive s'identifie à un bien-être factice. L'Isle au dragon décrit, sous une forme allégorique, les démarches entreprises par Godbout pour sensibiliser l'opinion publique à la convoitise innommable d'une compagnie américaine désirant transformer cet îlot en port de mer pour super-pétroliers. Tel un Michel Beauparlant qui tente par tous les moyens de secouer la torpeur des insulaires pour qu'ils résistent à la construction de Dépotoirs Atomiques Contrôlés (DAC), Jacques Godbout pourchasse les responsables du projet monstrueux. Il fonde un comité écologique et forme des groupes de pression qui envahissent les officines gouvernementales. Il n'hésite pas non plus, à l'image de Beauparlant, à expédier des télégrammes à toutes oreilles sympathisantes.

Ce Je de l'Isle au dragon, chasseur de dragons génial, qu'aucune censure ne retient, dénonce avec vivacité les Shaheen $\mathrm{Jr}$ qui veulent dominer grâce au pouvoir de l'argent, même si leur mépris barbare de l'environnement brise à tout jamais l'équilibre déjà fragile du milieu. Le désir humanitaire de Godbout, transposé ici dans la quête décidée du chasseur de dragons, aboutit au succès. L'ennemi, vaincu par la ruse et happé par le monstre de Cacouna, disparaît à jamais.

En somme, les narrateurs, sortes de doublures du Je, celui-là même de Godbout, écrivent et crient à haute voix ce qu'ils pensent de la société. De par la structure même du journal intime, le Je, agent de l'écriture, joue aussi le rôle d'un lecteur intradiégétique de son propre "manuscrit" en ce sens que, hypothétiquement, les François Galarneau, les Charles-François et les aùtres écrivent leur texte "quotidien» seulement pour leur propre satisfaction. Le narrateur jouit donc en même temps des attributs du narrataire parce qu'il ne destine alors sa narration à personne d'autre qu'à luimême35), ou à son double, comme dans les Têtes à Papineau où Charles peut lire «le récit sanguinaire sur son écran jumeau36». La lettre de l'Institut canadien d'orientation professionnelle destinée à Beauparlant 37 , celle de Jacques, frère de François, datée du 7 avril 195838, comme celle d'Andrée dans l'Aquarium39, toutes ces lettres et bien d'autres sont avant tout lues par le destinataire, le $J e$ écrivant et lecteur. 
À la fois narrateur et narrataire, le $J e$, en quelque sorte à l'abri des poursuites, formule avec autorité ses opinions. Lucide sur lui-même, le bicéphale des Têtes à Papineau, n'hésite pas à affirmer: «Nous avons toujours été, pour les scribouilleurs, un morceau de choix. Un sujet aussi inévitable que la problématique de la langue ou le pénible portrait de l'homme colonisé canadien-français 40 ». L'œil critique du narrateurnarrataire écorche les institutions, les personnalités, voire un écrivain com- me André Gide, "un drôle de zèbre qui écrit des phrases à pentures pour analyser ses sentiments, comme une vieille fille peureuse, des qui, des que, ça s'enchaîne comme des canards dans un stand de tir 41 ". Bien que teintés de subjectivité, les jugements qui émaillent les pseudo-journaux intimes de Godbout, dessinent avec force l'échelle des valeurs des Je.

Les $J e$ des romans de Godbout offrent l'image d'une évolution bien planifiée. L'auto-conscience accrue du Moi, de l'Aquarium au Moi double des Têtes à Papineau, n'a-t-il pas comme toile de fond un autre Je qui formule des propositions 42 , celui de l'auteur avouant: «(...) j'ai une écriture -très limitée. C'est une écriture qui est à la première personne 43 ".

Si les Je des romans de Godbout laissent percer d'une certaine façon les murmures d'une voix intérieure hantée par des événements qui ont touché de près l'auteur, le Journal d'hiver parle directement du Moi, celui de Godbout, même s'il affirme n'avoir jamais confié à personne ce qu'il pense réellement de son $\mathrm{Je}$. Ce texte brosse une espèce de tableau aussi bien de l'affaire Charron que du succès des Têtes à Papineau; du Canada de Pierre-Elliot Trudeau que des événements de Varsovie; de la réalisation de son film Monologue Nord-Sud44 que de son séjour à Boston et à New York pour présenter Deux épisodes dans la vie d'Hubert Aquin45: Bref, Jacques Godbout confie à son journal intime ses opinions et lève un peu le voile sur son intimité. Il parle de sa femme qu'il aime, de son fils Alain, de ses souvenirs de la "petite école», du secrétaire victorien sur lequel il couche ses idées dans sa maison de campagne tournée vers les États-Unis. En somme, le Journal d'hiver affiche l'image d'un Je, celui de Godbout, avec beaucoup moins de parcimonie que celui retenu par l'instance romanesque:

\section{Miroirs d'encre}

Enfin, l'analyse de l'autoreprésentation répercute avec originalité certains côtés du Je narrateur comme le souligne avec justesse André Belleau: «Le roman qui met en scène un écrivain accomplit une réitération et même un dédoublement de l'auteur46». Même si on ne peut ici faire mention de tous les niveaux, de tous les paliers du phénomène, il est important de relever certaines caractéristiques qui permettent de constater encore avec plus de force cette espèce de narcissisme du'Je collé à la technique même des œuvres godboutiennes.

Godbout aime dévoiler synthétiquement l'ensemble du récit dès les premières pages, sorte de reflet du dénouement. Les passages qui entretiennent l'anticipation et la découverte avant terme de la fin, renferment une 
capacité de rapprochement unissant, selon les termes de Ricardou, «deux cellules fictionnelles éloignées à partir de leur ressemblance 47 ». C'est ainsi que Lui de l'Aquarium, le personnage qui va mourir, prédit le dénouement: «Je vous annonce la domination, le massacre, la solitude. Je vous annonce le mépris d'un être qui vous fera ramper - vous deviendrez vermine à force de ramper 48 ". Cette façon de procéder, qui rend l'invisible visible ou le caché révélé, se produit aussi dans l'Isle au dragon où le narrateur, dès les premières pages, pressent déjà l'atmosphère du «combat final et sans témoins contre William T. Shaheen $\mathrm{Jr}_{\mathrm{r}} 4 \mathrm{y}_{\text {') }}$. Il en va de même des premières lignes des Têtes à Papineau où l'on apprend que l'aventure du bicéphale se solde par une intervention chirurgicale définitive. Les mécanismes retenus par l'auteur-narrateur dans les pseudo-journaux intimes s'astreignent à une sorte de mimétisme qui confond le point de départ et le point d'arrivée. La récurrence de cette "représentation intratextuelle, ou autoreprésentation 50 " telle que pratiquée par Godbout, ne peut que réveiller des soupçons concernant l'ascendance du $J e$ s'amusant avec les ficelles que le pacte autobiographique autorise.

En revanche, les premières pages du Couteau sur la table présentent le $J e$ narrateur qui, avant de situer l'histoire de sa liaison avec Patricia, anticipe sur les événements et pénètre, de force, dans un passé troublé. La plupart des incursions dans le passé trouvent leur explication dans le présent. Le présent réfléchit le passé et cette reduplication répétée entre l'instant présent et un autre moment déjà vécu semble justifier l'enchevêtrement constant des deux niveaux temporels. Le réglage de la mémoire, le rappel du passé suscité par Je, donne un sens à son agir, que ce soit avec Patricia ou avec Madeleine, de telle sorte que l'histoire d'amour est reléguée au second plan, car l'auteur désire insister avant tout sur la condition sociale du Canadien français. Je revoit son passé mais ne le vit pas. Ce qu'il vit, c'est son présent aux prises avec les complications sociales qu'éclairent les rencontres à l'Expresso Bar. Bien plus, le déroulement temporel annonce une fin où la mésentente germera. L'atmosphère répétitive temporelle engendre une lassitude telle que seule une "explosion" peut se produire, comme le note Jacques Allard51.

D'un autre côté, dans les Têtes à Papineau, l'intérêt soutenu que le double $J e$ attache au chiffre deux, comme le plaisir issu du «bis repetita placent», ressemble à ce que Ricardou appelle une "mise en abyme littérale52\%. Le chiffre deux et les nombres pairs organisent la toile de fond de ce roman. À partir des parents qui baignent dans «l'approche binaire53» jusqu'à Charles-François «monstre double autositaire54» et à sa sœur «Bébée», - récurrence phonétique de la deuxième lettre de l'alphabet -, en passant par tous les rappels de personnages où la correspondance relative au chiffre deux est exploitée, bref, la totalité de la similitude littérale du domaine numérique est organisée par une répétitions du nombre deux, qui suggère la bipartition cellulaire des cerveaux de Charles et de François, de même que leur double orientation distincte. Ces multiples redondances qui émaillent le récit des Têtes à Papineau, en plus d'établir d'une certaine 
manière la naissance de Charles et de François doublement conçus par Marie Lalonde, exposent une façon d'exprimer deux voix, deux têtes. La tête de l'un réfléchit l'autre, et vice versa. C'est comme le reflet propre au miroir.

Le mimétisme provoqué par les multiples ressemblances de la convenance chiffrée du nombre deux, et le redoublement de certaines instances discursives, transmettent une espèce de réflexivité que le système de dédoublement des mêmes lettres figure. Bien entendu, l'extension qui accompagne ce phénomène lexical n'est pas sans projeter un éclairage sur la signification à donner à ces répétitions. Ces récurrences, sortes de miroitements de la pensée de l'un dans l'agir de l'autre ou de l'action de l'un issue de l'idée de l'autre, sont l'image d'un Je tiraillé entre deux idéologies et qui, grâce aux reflets que lui retournent les événements quotidiens, s'auto-analyse.

Il est possible de prolonger notre réflexion sur les connivences cachées dans le dédoublement des personnages. Deux exemples parmi d'autres suffiront à illustrer ce procédé. Dans Salut Galarneau!, Jacques incite François à écrire, à parler de lui et de ce qui lui tient à coeur. Jacques est comme l'ombre dirigeante de son frère. En effet, depuis sa lettre du début; Jacques inspire directement François dans la confection de ses deux cahiers. Peu à peu, toutefois, François démystifie l'image de Jacques. François écrit donc son journal intime en voulant d'abord s'identifier à Jacques, mais par la suite, après maintes expériences, il redevient son propre maître à penser, il retrouve son Je. Certes, il n'est "pas un écrivain professionnel55», il se range plutôt du côté des artisans de la création. Bref, François représente une imagè «améliorée» de Jacques, un double se retrouvant en train d'écrire un livre dans lequel il se raconte lui-même.

Si la réverbération produite dans le cas de François et de Jacques améliore l'image du premier, la problématique de l'impossible unification du bicéphale Charles-François des Têtes à Papineau, la gémellité de leur entité et leur double caractère dessinent un portrait où le double $J e$, condamné à être toujours le vis-à-vis de l'autre, se mire dans son alter ego comme dans un miroir. La dimension référentielle que multiplient, pour ainsi dire, et les miroitements optiques qui sillonnent les écrans cathodiques et la parité Charles-François, abonde dans le sens de la thèse écrite par Charles sur «la mise en abîme (sic) du personnage des jumeaux dans le roman d'aventure ${ }^{56}$ ). La -composition même du bicéphale, sa structure intrinsèque, suggère l'image d'une unité qui se divise obligatoirement en miroitements réciproques, car les caractères, bien qu'opposés, se rejoignent dans une seule identité civile, un Je.

L'utilisation répétée du phénomène de la mise en abyme dans les romans-journaux intimes de Godbout ne permet-elle pas une «réflexion» brillamment entretenue favorisant le «caractère spécifique d'intimité qui le referme sur lui-même 57 », comme le note Jacques Rustin à propos du pseudo-journal intime? À l'instar des François, des Beauparlant ou des 
Papineau, l'écrivain Jacques Godbout aspire à l'autonomie que lui procure «l'invention patiente d'une écriture 58 ". Ce jumelage d'activités dans le procès d'énonciation résulte sans doute d'un processus narcissique conscient et, dirait-on, volontairement exploité beaucoup plus que d'une technique seulement bien assimilée. Godbout affirme, en effet: «Je suis éminemment conscient du dédoublement depuis ma tendre enfance. J'ai joué un personnage imaginaire quand j'étais tout jeune. J'ai continué. Me voyant faire des choses - moi agissant, moi ici vous parlant, et en même temps, moi regardant moi dans la caméra...59».

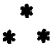

Ce qui identifie les Je de Godbout, aussi bien ceux des pseudojournaux intimes que du Journal d'hiver, pourrait se classer sous l'étiquette d'une sorte d'inquiétude ontologique que l'Histoire ne cesse d'alimenter. Le diariste Godbout rédigeant «un cahier dans lequel on s'étale comme en tombant sur la glace $60 »$, propose des $J e$ qui ne facilitent pas toujours la réduction de l'hiatus entre la distinction barthésienne de l'écrivant et de l'écrivain. Quoi qu'il en soit, comme l'écriture du commentaire cinématographique est une tâche fascinante pour le cinéaste, la rédaction d'un journal intime, réel ou pas, est une espèce de métamorphose qui fait dire à Godbout, parodiant la formule même de la transsubstantiation: «Ceci est mon corps, ceci est ma voix 61 ".

1. Le Journal intime du Jacques Godbout. Le Journal d'hiver. Texte diffusé du 9 au 13 août 1982 au réseau FM de Radio-Canada et lu par Marcel Sabourin. Cette émission a été réalisée par Yves Lapierre. L'écrit est publiê dans Liberté, no 148, août 1983, p. 11-63.

2. Alain Goulet, «La construction du moi par l'autobiographie, Si le grain ne meurt d'André Gide», Texte, no 1, 1982, p. 51.

3. Paul Toupin, Souvenirs pour demain, Montréal, CLF poche, no 9, 1968. À l'instar de Montaigne, Toupin écrit ses «Mémoires» pour lui-même: «Je suis le père légitime de ce que mes souvenirs ont d'authentique", dit-il (p. 30).

4. Béatrice Didier est d'avis que «l'identité auteur-narrateur-personnage peut être camouflée de façon beaucoup plus durable si l'auteur prête un nom au narrateur», le Journal intime, Paris, PUF, 1976, p. 149.

5. Ce terme est emprunté à Tzvetan Todorov, Littérature et Signification, Paris, Larousse, 1967, p. 83.

6. Gerard Genette, Figures III, Paris, Seuil, 1972, p. 229.

7. Salut Galarneau!, Paris, Seuil, 1967.

8. Les Têtes à Papineau, Paris, Seuil, 1981.

9. Gerald Prince soutient avec à propos que: «The diary novel is a roman personnel, an IchRoman, a first-person novel in which the narrator is a protagonist in the events he records", "The Diary Novel: Notes for the Definition of a Sub-Genre", Neophilologus, LIX, no 4, octobre 1975, p. 477.

10. Jean Rousset, Narcisse romancier, Paris, Librairie José Corti, 1973, p. 57.

11. Gérard Genette, Palimpsestes, Paris, Seuil, 1982, p. 89.

12. Journal d'hiver, vendredi, 25 décembre 1981.

13. Ibid., vendredi, 23 avril 1982. 
14. Ibid., mercredi, 3 février 1982.

15. Peter Boerner, «Place du journal», le Journal intime et ses formes littéraires, GenèveParis, Librairie Droz, 1978, p. 222.

16. L'Aquarium, Paris, Seuil, 1962.

17. Le Couteau sur la table, Paris, Seuil, 1965.

18. L'Isle au dragon, Paris, Seuil, 1976.

19. Les Têtes à Papineau, p. 28.

20. Ibid., p. 64.

21. Cf. Béatrice Didier, op. cit, p. 188.

22. D'amour, P.Q., Paris-Montréal, Seuil-Hurtubise HMH, 1972.

23. Gilles Marcotte, le Roman à l'imparfait, Montréal, La Presse, 1976, p. 150-151.

24. Salut Galarneau!, p. 105-106.

25. L'Aquarium, p. 127.

26. Ibid., p. 13-17.

27. Ibid., p. 61.

28. Ibid., p. 42.

29. Président du Mouvement laïque de langue française de 1966 à 1968, Jacques Godbout publie alors un manifeste qui résume sans détours sa pensée sur la confessionnalité et l'Église en général. Le Mouvement du 8 avril, Montréal, collection MLF, 28 p., reproduit dans le Réformiste. Textes tranquilles, Montréal, AS Quinze, p. 91-102. On peut lire aussi la note sur Gilles Gascon, trésorier du M.L.F., mort à Paris en 1981, Journal d'hiver, samedi, 9 janvier 1982.

30. R. Robidoux, «l'Aquarium de Jacques Godbout», le Roman canadien-français du XXe siècle, Ottawa, Éditions de l'Université d'Ottawa, coll. Visage des lettres canadiennes, 1966, p. 201.

31. Journal d'hiver, lundi, 28 décembre 1981.

32. Dans la nuit du 20 au 21 avril 1963, le Front de libération du Québec avait placé une bombe derrière le centre de recrutement de l'armée canadienne rue Sherbrooke à Montréal. L'engin a tué le veilleur de nuit, Wilfrid O'Neil, victime innocente. Cette violence qui frappe inconsidérément a changé la vie de Godbout et aussi la fin du roman.

33. Marie-Claire Blais, Une saison dans la vie d'Emmanuel, Montréal, Éditions du Jour, 1965.

34. Journal d'hiver, vendredi, 25 décembre 1981.

35. Gerald Prince, "Introduction à l'étude du narrataire", Poétique, no 14, 1973, p. 188.

36. Les Têtes à Papineau, p. 146.

37. L'Isle au dragon, p. 81-83.

38. Salut Galarneau!, p. 15-19.

39. L'Aquarium, p. 93-95.

40. Les Têtes à Papineau, p. 27.

41. Salut Galarneau!, p. 81.

42. Journal d'hiver, mercredi, 3 février 1982.

43. J. Godbout, "Entrevue», Québec français, no 26, mai 1977, p. 30.

44. Un Monologue Nord-Sud (film), 57 min., $40 \mathrm{sec}$., C., ONF, 1982.

45. Deux épisodes dans la vie d'Hubert Aquin (film), $56 \mathrm{~min} ., 50 \mathrm{sec}$., C., ONF, 1979.

46. André Belleau, le Romancier fictif, Québec, P.U.Q., 1980, p. 22-23.

47. Jean Ricardou, «L'escalade de l'autoreprésentation», Texle, no 1, 1982, p. 16.

48. L'Aquarium, p. 33.

49. L'Isle au dragon, p. 15.

50. Jean Ricardou, Nouveaux Problèmes du roman, Paris, Éditions du Seuil, 1978, p. 104. 


\section{VOIX ET IMAGES, VOL. X, NO 3 (PRINTEMPS 85)}

51. Jacques Allard, «I, ni, mi, ni, maï, ni, mo», dans Parti pris, août-septembre 1965, p. 112.

52. Jean Ricardou, op. cit., p. 159.

53. Les Têtes à Papineau, p. 109.

54. Ibid., p. 131.

55. Salut Galarneau!, p. 129.

56. Les Têtes à Papineau, p. 111.

57. Jacques Rustin, «La Religieuse de Diderot: mémoires ou journal intime? », le Journal intime et ses formes littéraires, Genève-Paris, Librairie Droz, 1978, p. 33.

58. J. Godbout, le Reformiste. Textes tranquilles, Montréal, AS Quinze, 1975, p. 183.

59. J. Godbout, «Entrevue», Québec français, no 26, mai 1977, p. 31.

60. Salut Galarneau!, p. 155.

61. Journal d'hiver, lundi, 11 janvier 1982. C'est nous qui soulignons. 